\title{
Chimaeras reveal the role of the catalytic core in the activation of the plasma membrane $\mathrm{Ca}^{2+}$ pump
}

\author{
William BA-THEIN*1, Ariel J. CARIDE*, Ágnes ENYEDI†, Katalin PÁSZTY†, Cynthia L. CROY*, Adelaida G. FILOTE0* \\ and John T. PENNISTON*2 \\ ${ }^{*}$ Department of Biochemistry and Molecular Biology, Mayo Foundation, 200 First Street South West, Rochester, MN 55905, U.S.A., and †National Institute of \\ Haematology and Immunology, Daroczi utca 24, 1113 Budapest, Hungary
}

Isoform $2 \mathrm{~b}$ of the plasma membrane calcium pump differs from the ubiquitous isoform $4 \mathrm{~b}$ in the following: (a) higher basal activity in the absence of calmodulin; (b) higher affinity for calmodulin; and (c) higher affinity for $\mathrm{Ca}^{2+}$ in the presence of calmodulin [Elwess, Filoteo, Enyedi and Penniston (1997) J. Biol. Chem. 272, 17981-17986]. To investigate which parts of the molecule determine these kinetic differences, we made four chimaeric constructs in which portions of isoform $2 \mathrm{~b}$ were grafted into isoform $4 \mathrm{~b}$ : chimaera I contains only the C-terminal regulatory region of isoform $2 \mathrm{~b}$; chimaera II contains the $\mathrm{N}$ terminal moiety of isoform $2 \mathrm{~b}$, including both cytoplasmic loops; chimaera III contains the sequence of isoform $2 \mathrm{~b}$ starting from the N-terminus to after the end of the first (small) cytoplasmic loop; and chimaera IV contains only the second (large) cytoplasmic loop. Surprisingly, chimaera I showed low basal activity in the absence of calmodulin and low affinity for calmodulin, unlike isoform $2 \mathrm{~b}$. In contrast, the chimaera containing both loops showed high basal activity, and $\mathrm{Ca}^{2+}$ activation curves (both in the absence and in the presence of calmodulin) similar to those of isoform $2 \mathrm{~b}$. The rates of activation by calmodulin and of inactivation by calmodulin removal were measured, and the apparent $K_{\mathrm{d}}$ for calmodulin was calculated from the ratio between these rate constants. The order of affinity was: $2 b=\mathrm{II}>4 \mathrm{~b}=$ $\mathrm{IV}>\mathrm{III}=\mathrm{I}$. From these results it is clear that the construct that most closely resembles isoform $2 \mathrm{~b}$ is chimaera II. This shows that, in order to obtain an enzyme with properties similar to those of isoform $2 b$, both cytoplasmic loops are needed.

Key words: calmodulin, $\mathrm{Ca}^{2+}$ signal, ion transport, P-type ATPase.

\section{INTRODUCTION}

The plasma membrane calcium pump (PMCA) actively transports $\mathrm{Ca}^{2+}$ across the plasma membrane, and is necessary for control of the calcium concentration inside the cell. At low cytoplasmic $\mathrm{Ca}^{2+}$ concentrations, this pump is maintained in an inactive state by the interaction of its catalytic core with an autoinhibitory region in the C-terminus of the molecule. As discussed previously [1], activation of PMCA is accomplished by the disruption of this interaction by calmodulin. A study in which the properties of the rat PMCA2b and human PMCA4b isoforms were compared suggested that differences in the sequences of these molecules can influence this interaction. The basal activity (measured in the absence of calmodulin) of PMCA2b is much greater than that of PMCA4b, and the apparent calmodulin affinity is also about 4-fold higher [2]. Previous studies [3] using synthetic peptides representing the calmodulin-binding domains of different $b$ isoforms have indicated that the small differences in their calmodulin-binding domains cannot be responsible for the discrepancy in their calmodulin affinity. Rather, this discrepancy comes from the sequence variations in either the catalytic core or the C-terminus downstream of the calmodulinbinding domain, or both. This indicates that further comparisons between isoforms $2 \mathrm{~b}$ and $4 \mathrm{~b}$ may shed light on the intramolecular interaction of the core with the C-terminus.

Predictions of the structure of PMCA, based on the amino acid sequence [4] and on structural data for the related sarcoplasmic reticulum $\mathrm{Ca}^{2+}$ pump [5], reveal that the cytoplasmic side of the $\mathrm{Ca}^{2+}$ pump contains two loops. These loops are predicted to form the site for catalysis of the hydrolysis of ATP. We therefore made several chimaeras of these two isoforms, in which we replaced portions of PMCA $4 \mathrm{~b}$ with the corresponding regions of isoform $2 b$ (see Figure 1). The results reported here show that the regulatory $\mathrm{C}$-terminal region interacts with these two loops. They also show that exchanges of the loops (included in the region Met-1-Arg-704) between isoforms 2b and 4b are important in determining the strength of its interaction with the regulatory region, while exchanges of the regulatory $\mathrm{C}$-terminal regions (Ile-1100 to Val-1205) are less important.

\section{MATERIALS AND METHODS}

\section{Materials}

${ }^{45} \mathrm{Ca}$ and $\left[\gamma_{-}{ }^{32} \mathrm{P}\right] \mathrm{ATP}$ were purchased from DuPont NEN. Calmodulin was obtained from Sigma. LIPOFECTAMINE ${ }^{\mathrm{TM}}$ and Optimem media were obtained from Gibco BRL Life Technologies, Inc. All other chemicals used were of reagent grade. Rat isoform $2 b$ and human isoform $4 b$ were used in all experiments. The sequences of the corresponding rat and human isoforms of PMCA are nearly identical.

\section{Chimaera construction}

PMCA isoform $4 \mathrm{~b}$, cloned into the $S a l I$ and $K p n I$ sites of pSP72, and PMCA isoform 2bz, cloned into the SalI and KpnI sites of the PMM2 expression vector, served as starting points for chimaera construction. The unique restriction enzyme sites ScaI, $S t u \mathrm{I}, S m a \mathrm{I}$ and BamHI in isoform $4 \mathrm{~b}$ provided points at which

Abbreviation used: PMCA, plasma membrane $\mathrm{Ca}^{2+}$ pump

1 Present address: Department of Microbiology, University of Tsukuba, Tsukuba 305-8578, Japan

2 To whom correspondence should be addressed (e-mail penniston.john@mayo.edu). 


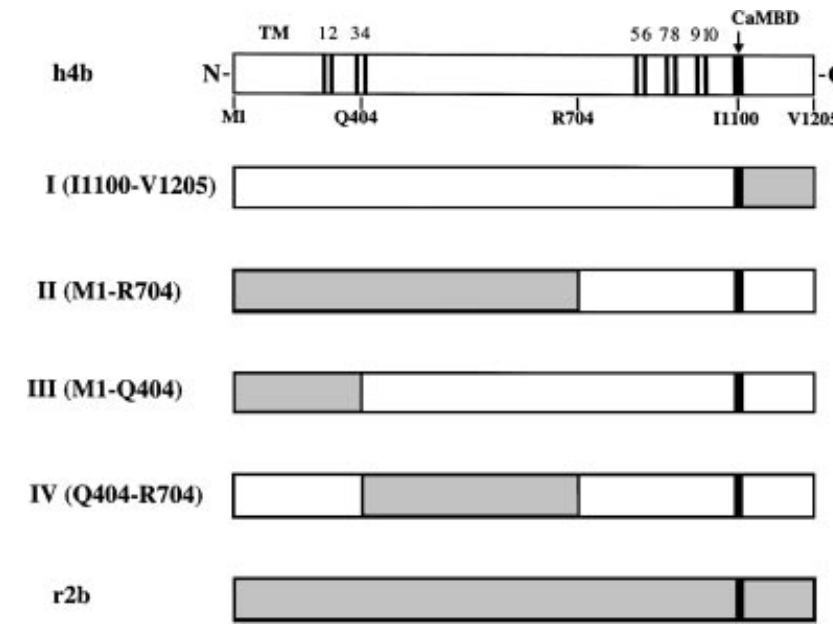

Figure 1 Chimaeras used in the present study

The 10 transmembrane (TM) regions are indicated by the thin vertical lines, which are numbered to identify them. 'CaMBD' refers to the calmodulin-binding domain (which does not pass through the membrane). Substitution of regions of isoform $2 b$ for the corresponding regions of isoform $4 \mathrm{~b}$ is indicated by grey shading of the region. Note that chimaera II (Met1-Arg-704) contains both cytoplasmic loops, while chimaera III (Met-1-Gln-404) contains the small cytoplasmic domain, and chimaera IV (Gln-404-Arg-704) contains the large cytoplasmic domain. The prefixes $\mathrm{h}$ and $\mathrm{r}$ denote human and rat respectively.

\section{Table 1 Primers used to make the chimaeras}

Nucleotides that introduce mutations are shown italicized and in bold. New restriction endonuclease sites are underlined.

\begin{tabular}{ll}
\hline Primer & Sequence \\
\hline A & atc ccc gag gaa gag tt \\
B & cca ggc gac agg tac $\boldsymbol{c}$ \\
C & gca agc cta tgt cgg tga tgt cc \\
D & ggg cag gcc $t c c$ ctc tt tt \\
E & ggt gct tca ggg caa gct \\
F & aag agg gag $\mathbf{g c c}$ tgc c \\
G & ggt gga taa tgc cac act t
\end{tabular}

to substitute segments of isoform $4 \mathrm{~b}$ with those of isoform $2 \mathrm{bz}$. These sites correspond to amino acids Gln-404, Gly-526, Arg704 and Ile-1100 respectively.

Chimaera I did not require any intricate manipulations. Both isoforms $4 \mathrm{~b}$ and $2 \mathrm{bz}$ have a Bam HI site at corresponding locations. Primer A (Table 1) extends through this site. Primer B introduces a $K p n \mathrm{I}$ site. While isoform 2 bz already had a $K p n \mathrm{I}$ site after the stop codon, it also had a substantial 3' untranslated region. Addition of the $K p n I$ site to primer B eliminates the 3' untranslated region that the original $K p n I$ site would have introduced into chimaera I. Construction of chimaera II was straightforward, because isoform $2 \mathrm{bz}$ shares the SmaI site at a corresponding position. Thus a simple replacement of the SalI/SmaI fragment in $4 \mathrm{~b}$ with the corresponding fragment from $2 \mathrm{bz}$ created chimaera II.

Chimaera III was more challenging, because the ampicillin resistance gene has a $S c a I$ site. Therefore it was not possible to directly replace the $S a l \mathrm{I} / \mathrm{ScaI}$ fragment in pSP72, even though the $S c a I$ sites in isoforms $2 \mathrm{bz}$ and $4 \mathrm{~b}$ occur at corresponding points. This dilemma was resolved by destroying the $S c a$ I site in
pSP72 containing isoform 4b. A 1340 bp StuI/StuI fragment from the pACYC177 vector, which contains the kanamycin resistance gene, was blunt-cloned into the $S c a$ I site of pSP72. This vector is designated $\mathrm{pSP} 72 ; \mathrm{Kn}$. The $\mathrm{SalI} / \mathrm{ScaI}$ replacement was then made directly and selected using kanamycin resistance.

Chimaera IV was the most challenging, because the kanamycin resistance gene has a SmaI site. Therefore neither the native nor the modified pSP72 vector could be used for a direct replacement of the ScaI/SmaI fragment. Therefore this chimaera was constructed from the Gln-404-Gly-526 and Gly-526-Arg-704 chimaeras (not shown). These constructs took advantage of the unique $S t u \mathrm{I}$ site in isoform $4 \mathrm{~b}$. However, isoform $2 \mathrm{bz}$ does not share a $S t u \mathrm{I}$ site at the corresponding point; it has a StuI site 140 nucleotides upstream from the corresponding position in isoform 4b. A megaprimer PCR approach was used to construct the Gln404-Gly-526 chimaera. Primers C and D were used with isoform $2 \mathrm{bz}$ as template to create the megaprimer. This primer destroyed the native $S t u \mathrm{I}$ site in isoform $2 \mathrm{bz}$ and added a StuI site at a position corresponding to that in isoform $4 \mathrm{~b}$. Primer $\mathrm{E}$ and the megaprimer were used with isoform $2 \mathrm{bz}$ as template to create a product with the $S c a \mathrm{I} / S t u \mathrm{I}$ fragment needed for this chimaera. This product was cloned into the pCR-Blunt II vector, and the replacement of the $S c a \mathrm{I} / S t u \mathrm{I}$ fragment in the kanamycinresistant isoform $4 \mathrm{~b}$ was carried out using standard molecular biology techniques. This construct was then transferred to the native pSP72 vector in preparation for making chimaera IV. Primer F introduces a StuI site in isoform $2 \mathrm{bz}$ at the same position as primer $\mathrm{C}$. Primers $\mathrm{F}$ and $\mathrm{G}$ with isoform $2 \mathrm{bz}$ as template created a StuI/SmaI fragment. Creation of the Gly526-Arg-704 chimaera only required replacing the StuI/SmaI segment of isoform $4 \mathrm{~b}$ with this fragment. Also, with this fragment, creation of chimaera IV was a simple matter of replacing the StuI/SmaI fragment of the Gln-404-Gly-526 chimaera cloned into native pSP72. It should be noted that the mutations introduced by primers $\mathrm{D}$ and $\mathrm{F}$ are not silent in isoform 2b. Therefore Gly-526 was left instead of the corresponding Ala from isoform $2 b$. Hence chimaera IV contains the large cytoplasmic loop of PMCA2, with an $A \rightarrow G$ substitution at position 526. This has to be taken into account when interpreting the results.

\section{Transfection of COS-1 cells}

Transfection was carried out using LIPOFECTAMINE ${ }^{\text {TM }}$ (Gibco BRL Life Technologies, Inc.), based on the protocol described by the manufacturer and in Enyedi et al. [6]. Briefly, transfection was initiated when the cells were $70-80 \%$ confluent in $150 \mathrm{~cm}^{2}$ flasks. The cells were incubated at $37^{\circ} \mathrm{C}$ with the DNALIPOFECTAMINE ${ }^{\text {TM }}$ complex (formed by incubating $8 \mu \mathrm{g}$ of DNA and $100 \mu 1$ of LIPOFECTAMINE ${ }^{\text {TM }}$ in $3.6 \mathrm{ml}$ of serum-free Optimem medium) in $14.5 \mathrm{ml}$ of serum-free Optimem medium. After a $5 \mathrm{~h}$ incubation, the cells were supplemented with serum, and incubation was continued for a total of $24 \mathrm{~h}$. The medium containing the DNA-LIPOFECTAMINE ${ }^{\mathrm{TM}}$ complex was then replaced with fresh tissue culture medium containing $10 \%(\mathrm{v} / \mathrm{v})$ serum, and the cells were cultured for an additional $24 \mathrm{~h}$.

\section{Isolation of microsomes from COS cells}

Crude microsomal membranes from COS cells were prepared as described by Enyedi et al. [7].

\section{$\mathrm{Ca}^{2+}$ transport assay}

$\mathrm{Ca}^{2+}$ uptake by microsomal vesicles was carried out in a $200 \mu 1$ reaction mixture and assayed by rapid filtration through 
Millipore membrane filters $(0.45 \mu \mathrm{m}$ pore size; type HA) as described previously [6,7]. The reaction mixture contained $100 \mathrm{mM} \mathrm{KCl}, 25 \mathrm{mM}$ Tes/triethanolamine, $\mathrm{pH} 7.2,40 \mathrm{mM}$ $\mathrm{KH}_{2} \mathrm{PO}_{4} / \mathrm{K}_{2} \mathrm{HPO}_{4}$, pH 7.2, $200 \mathrm{nM}$ thapsigargin, $5 \mathrm{mM} \mathrm{NaN}_{3}$, $4 \mu \mathrm{g} / \mathrm{ml}$ oligomycin, $7 \mathrm{mM} \mathrm{MgCl} \mathrm{M}_{2}, 100 \mu \mathrm{M} \mathrm{CaCl}_{2}$ (labelled with ${ }^{45} \mathrm{Ca}$; specific radioactivity $100000-150000$ c.p.m./nmol) and enough EGTA to obtain the desired free $\mathrm{Ca}^{2+}$ concentration. Microsomes at $10-20 \mu \mathrm{g} / \mathrm{ml}$ concentration were preincubated in the presence or absence of $2 \mu \mathrm{M}$ calmodulin for $2 \mathrm{~min}$ at $37^{\circ} \mathrm{C}$, and $\mathrm{Ca}^{2+}$ uptake by the vesicles was started by the addition of $6 \mathrm{mM}$ ATP. The reaction was terminated by rapid filtration of the microsomes using Millipore membrane filters.

\section{Determination of $\boldsymbol{k}_{\mathrm{on}}$ and $\boldsymbol{k}_{\mathrm{off}}$ for activation by calmodulin}

These constants were measured in experiments in which activation by calmodulin of $\mathrm{Ca}^{2+}$-ATPase activity, and its inactivation by calmodulin removal, were measured. This is described in detail in [8]. The kinetic method used to measure ATPase activity was also described in that paper [8]. $\mathrm{Ca}^{2+}$ ATPase activity was measured in medium containing $30 \mathrm{mM}$ Tes/triethanolamine (pH 7.2), $120 \mathrm{mM} \mathrm{KCl}, 5 \mathrm{mM} \mathrm{MgCl}_{2}$, $200 \mu \mathrm{M}$ EGTA, $0.2 \mathrm{mM}$ 2-amino-6-mercapto-7-methylpurine, $1 \mathrm{unit} / \mathrm{ml}$ purine nucleoside phosphorylase, $5 \mathrm{mM} \mathrm{NaN}_{3}, 1 \mathrm{mM}$ dithiothreitol, $0.5 \mathrm{mM}$ ouabain, $4 \mu \mathrm{g} / \mathrm{ml}$ oligomycin, $200 \mathrm{nM}$ thapsigargin, $2 \mu \mathrm{g} / \mathrm{ml}$ aprotinin, $0.5 \mu \mathrm{g} / \mathrm{ml}$ leupeptin and enough $\mathrm{CaCl}_{2}$ to obtain $0.4 \mu \mathrm{M}$ free $\mathrm{Ca}^{2+}$.

\section{RESULTS}

Out of 28 residues, 26 are identical between the calmodulinbinding domains of PMCA isoforms $2 b$ and $4 b$. Despite the great similarity in their calmodulin-binding domains, the apparent calmodulin affinity of isoform $2 \mathrm{~b}$ is approx. 4 times higher than that of isoform $4 \mathrm{~b}[2]$.

To test whether the differences between isoforms $2 b$ and $4 b$ are due to their C-termini, we made a chimaera (I) which has the Cterminal regulatory region of isoform $2 b$ replacing that of isoform $4 \mathrm{~b}$. Figure 2 shows that the apparent calmodulin affinity of chimaera I was even lower than that of isoform $4 \mathrm{~b}$, indicating

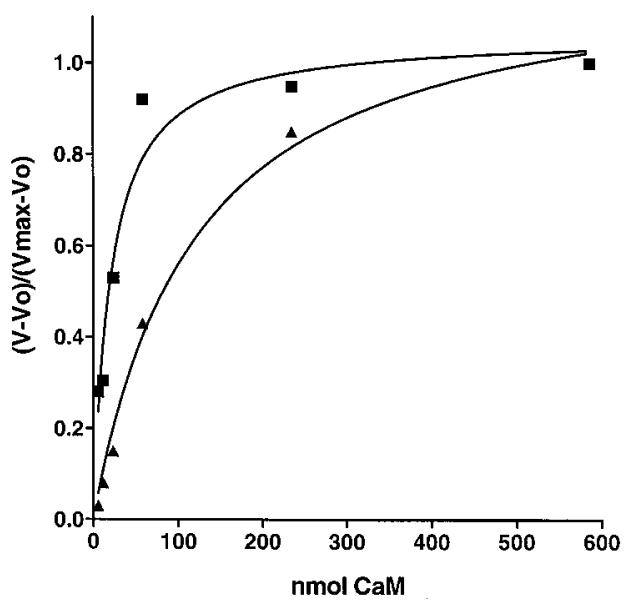

Figure 2 Effects of calmodulin on calcium uptake by PMCA4b and chimaera I

Calcium transport was measured in COS cell microsomes expressing isoform $4 \mathrm{~b}(\boldsymbol{\square})$ or chimaera I ( $\mathbf{\Delta})$, as described in the Materials and methods section. $V_{0}$ is the residual activity in the absence of calmodulin, $V \max$ is the maximal activity, and $V$ is the observed activity at a given calmodulin concentration.

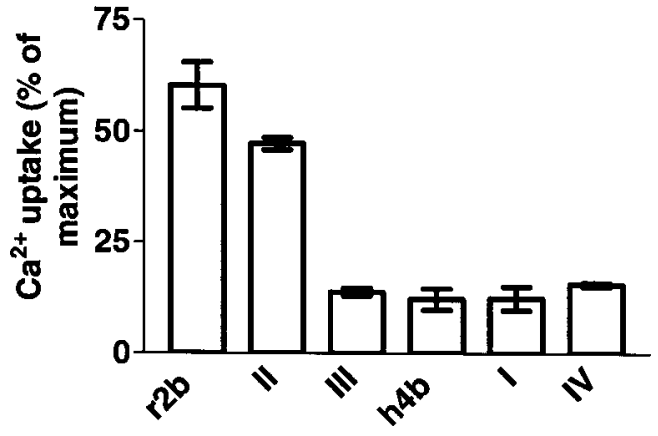

Figure $3 \mathrm{Ca}^{2+}$ uptake by PMCA isoforms $4 \mathrm{~b}$ and $2 \mathrm{~b}$ and by the various chimaeras in the absence of calmodulin

The conditions used were as described in the legend of Figure 2. The prefixes $h$ and $r$ denote human and rat respectively.

that differences in the sequence of the $\mathrm{C}$-terminus (including the calmodulin-binding domain) cannot be responsible for the high calmodulin affinity observed for isoform $2 b$.

We then made several more chimaeras in which various portions of isoform $4 \mathrm{~b}$ were replaced by the corresponding regions of isoform $2 b$. Figure 1 shows the chimaeras used in the study, along with the exact portion of the isoform $4 \mathrm{~b}$ molecule that was replaced by portions of isoform $2 \mathrm{~b}$. Chimaera II contains a large portion of the N-terminal region of isoform $2 b$, including both of the cytoplasmic loops. Previous cross-linking studies suggested that both of these loops are involved in the interaction with the regulatory region of isoform $4 \mathrm{~b}$ [9]. Chimaera III contains the sequence of isoform $2 b$ starting from the $\mathrm{N}$ terminus to after the end of the first (small) cytoplasmic loop; chimaera IV contains only the second (large) cytoplasmic loop.

In order to assess the properties of the chimaeras, we began by measuring their basal activities. We already knew that isoform $2 \mathrm{~b}$ has a much higher basal activity than isoform $4 \mathrm{~b}$, indicating a weaker intramolecular interaction between the C-terminal regulatory region and the catalytic core. Figure 3 shows the activities of each of the chimaeras in the absence of calmodulin. It is clear that only chimaera II (containing both cytoplasmic loops) had a basal activity approaching that of isoform $2 b$, while the other chimaeras were indistinguishable from isoform $4 \mathrm{~b}$. This shows the necessity of the presence of both cytoplasmic loops of isoform $2 b$ to obtain higher basal activity. Our results suggest that both cytoplasmic loops are involved in determining the strength of the intramolecular interaction.

Reinforcing this point, Figure 4 shows the dependence of the activity on $\mathrm{Ca}^{2+}$ concentration for each of the chimaeras. Each activity is shown in the presence and in the absence of calmodulin. The chimaeras naturally divide into two groups, those that have a low basal activity like isoform $4 b$ (chimaeras I, III and IV) and that which has a high basal activity like isoform $2 \mathrm{~b}$ (chimaera II). The data in Figure 4 demonstrate that the results of the experiment in Figure 3 are valid at all $\mathrm{Ca}^{2+}$ concentrations observed here.

Another crucial parameter that differentiates isoform $2 \mathrm{~b}$ from isoform $4 \mathrm{~b}$ is the affinity for calmodulin. In Figure 5 the on and off rates for activation by calmodulin of isoforms $2 \mathrm{~b}$ and $4 \mathrm{~b}$ and the chimaeras are shown. The on-rate constants (Figure 5, top panel) can be classified in the following order: $2 \mathrm{~b}=\mathrm{II}>4 \mathrm{~b}=$ IV $>$ III $=$ I. The off-rate constants (Figure 5, middle panel) were in the following order: III $>4 \mathrm{~b} \sim \mathrm{IV}>2 \mathrm{~b}=\mathrm{I}=\mathrm{II}$. The $k_{\text {off }} / k_{\text {on }}$ ratio is the $K_{\mathrm{d}}$ for calmodulin for each of the constructs. 


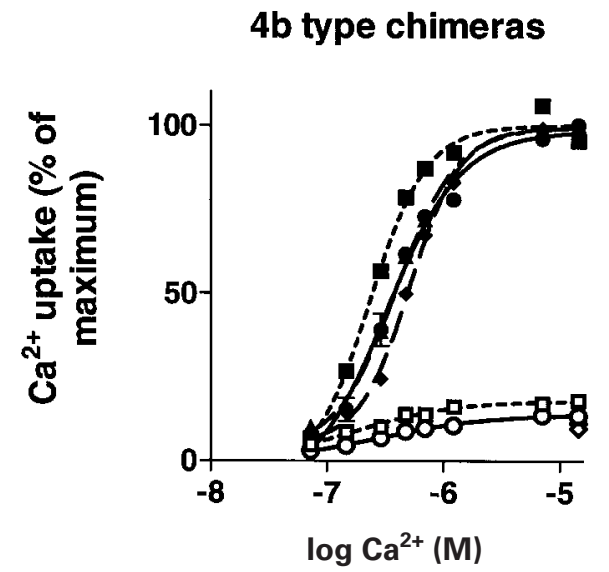

$2 b$ type chimeras

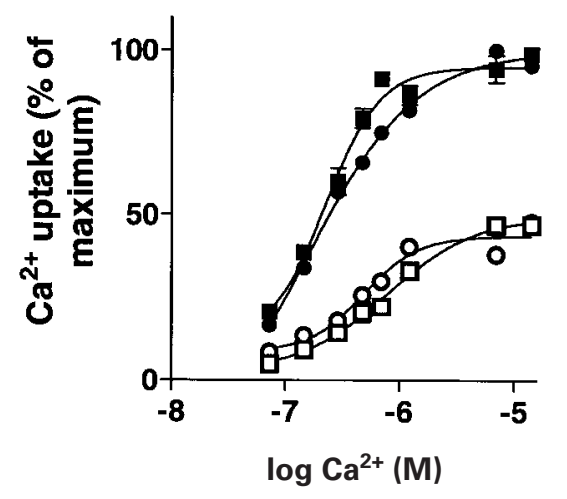

Figure $4 \mathrm{Ca}^{2+}$-dependence of activities of full-length PMCA isoforms $4 \mathrm{~b}$ and $2 \mathrm{~b}$, and of the chimaeras containing each of the cytoplasmic loops

The filled symbols show the activity in the absence of calmodulin, and the open symbols that in the presence of calmodulin. Measurements in the absence of calmodulin were made with chimaeras I and IV only at the lowest and highest $\mathrm{Ca}^{2+}$ concentrations. All measurements on other constructs covered a full set of $\mathrm{Ca}^{2+}$ concentrations. The conditions used were as described in the legend of Figure 2. Upper panel: $\boldsymbol{O}$, isoform $4 b+$ calmodulin; $\bullet$, chimaera I+ calmodulin; $\mathbf{\square}$, II + calmodulin; $\boldsymbol{\Delta}$, IV + calmodulin; $\bigcirc$, 4b minus calmodulin; $\diamond, I$ minus calmodulin; $\square$, III minus calmodulin; X, IV minus calmodulin (this symbol is obscured by the open circle symbol in the lower right-hand corner of the Figure). Lower panel: 2b + calmodulin; $\mathbf{\square}$ II + calmodulin; $\bigcirc$, 2b minus calmodulin; $\square$, II minus calmodulin.

Thus the calculated $K_{\mathrm{d}}$ values for isoform $2 \mathrm{~b}$ and chimaera II are very low, while isoform $4 \mathrm{~b}$ and chimaera IV have higher $K_{\mathrm{d}} \mathrm{s}$, and chimaeras I and III have the highest values. It is noticeable that the order of $K_{\mathrm{d}}$ values for the different chimaeras approximately reflects the distribution of basal activities, i.e. the constructs with the lowest $K_{\mathrm{d}}$ values have the highest basal activity (see Figure 3).

\section{DISCUSSION}

The goal of this work was to study the role of the C-terminus and the cytoplasmic loops in the regulation of PMCA2b and PMCA $4 b$ by calmodulin. Early studies indicated that calmodulin binding to the C-terminal region of PMCA activates the pump by preventing this region from interacting with the cytoplasmic core of the enzyme. The discovery of the existence of several isoforms of PMCA was followed by the finding that these isoforms differ in the properties of their activation by calmodulin [2,10-12]. Differences between isoforms $2 b$ and $4 b$ were especially striking: PMCA2b has a higher affinity for calmodulin and
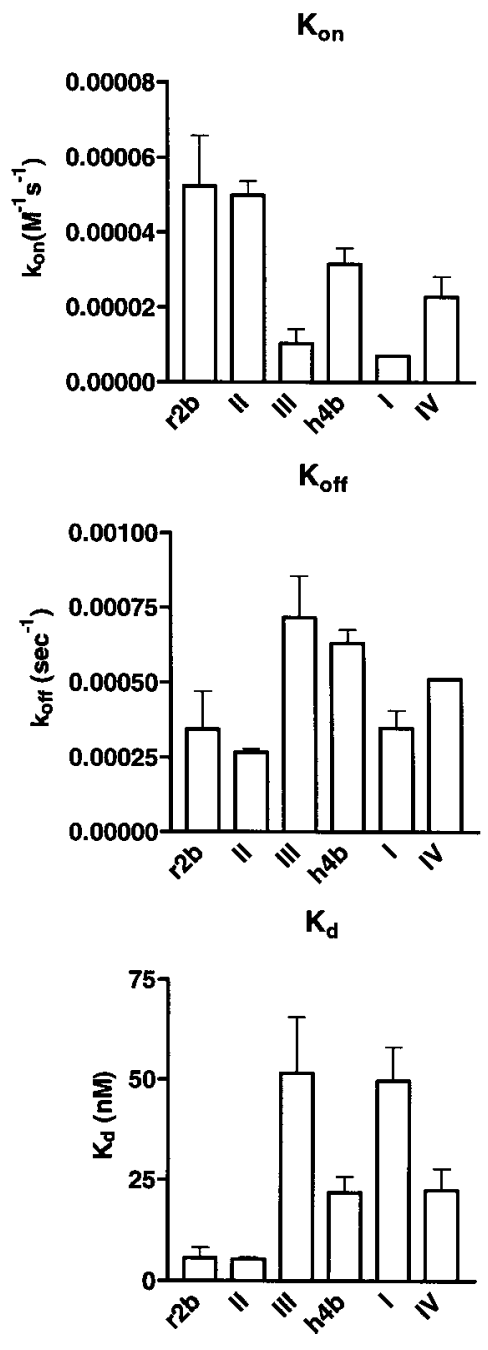

Figure 5 Analysis of the on and off rates for calmodulin binding to PMCA isoforms $4 \mathrm{~b}$ and $2 \mathrm{~b}$ and chimaeras I-IV

The rates of activation and inactivation, along with the overall $K_{\mathrm{d}}$, were determined from ATPase activity assays, as described in the Materials and methods section. The prefixes $h$ and $r$ denote human and rat respectively.

higher basal activity in calmodulin's absence than isoform $4 \mathrm{~b}$. The differences in primary structure between the two isoforms are located mainly in the C-terminal region of the pump, downstream of the calmodulin-binding domain. Furthermore, calmodulin-binding peptides having the sequence of either PMCA2b or PMCA4b bind calmodulin equally well with very high affinity. Because of this, we tried to explain the different behaviour of these isoforms on the basis of the differences between their sequences in the C-terminus, downstream of the calmodulin-binding domain. However, chimaera I (made up of the isoform $4 b$ core and the C-terminus of isoform $2 b$ ) had the same basal activity as and even lower calmodulin affinity than isoform $4 \mathrm{~b}$, which is the opposite of what we expected. From these data it is evident that the regions responsible for the differences in the response to calmodulin between isoforms $4 \mathrm{~b}$ and $2 \mathrm{~b}$ lie somewhere else. Of all the chimaeras that we prepared, only chimaera II (containing both cytoplasmic loops of isoform $2 b)$ had a calmodulin activation pattern like that of isoform $2 b$. Its basal activity, $K_{\mathrm{d}}$ for calmodulin and $\mathrm{Ca}^{2+}$ activation kinetics 
(both with and without calmodulin) are similar to those of isoform 2b. On the other hand, chimaeras III (containing only the small cytoplasmic loop of isoform $2 b$ ) and IV (containing only the large cytoplasmic loop of isoform $2 b$ ) have a calmodulin activation pattern similar to that of isoform $4 \mathrm{~b}$.

Despite binding calmodulin with extremely high affinity, the calmodulin-binding domain by itself does not determine the apparent affinity for calmodulin's activation of the pump. In fact, there are two orders of magnitude difference between the apparent affinities for calmodulin activation of PMCA isoform $2 \mathrm{~b}$ and the $K_{\mathrm{d}}$ for binding of calmodulin to C28R2, a calmodulinbinding peptide prepared based on the sequence of PMCA2b [3]. This observation reinforces the concept that activation by calmodulin is a complex process involving the interaction of several domains in the pump.

Summarizing, the present results are consistent with the idea that both cytoplasmic loops of PMCA are involved in the intramolecular interaction with the $\mathrm{C}$-terminal region that is disrupted upon binding of calmodulin. Our results are in agreement with those of the Carafoli laboratory [9], who, based on chemical cross-linking experiments, proposed that the calmodulin-binding domain interacts with two regions in the pump: one in the small cytoplasmic loop and the other in the large one.

This work was supported in part by National Institutes of Health grants GM28835 and GM55514 to J. T. P., by Hungarian Academy of Sciences grant OTKA T023659 to Á. E., and by an International Research Scholarship from the Howard Hughes Medical Institute (Á. E. and J. T. P.)

\section{REFERENCES}

1 Penniston, J. T. and Enyedi, Á. (1998) Modulation of the plasma membrane $\mathrm{Ca}^{2+}$ pump. J. Membr. Biol. 165, 101-109
2 Elwess, N. L., Filoteo, A. G., Enyedi, A. and Penniston, J. T. (1997) Plasma membrane $\mathrm{Ca}^{2+}$ pump isoforms $2 \mathrm{a}$ and $2 \mathrm{~b}$ are unusually responsive to calmodulin and $\mathrm{Ca}^{2+}$. J. Biol. Chem. 272, 17981-17986

3 Enyedi, Á., Filoteo, A. G., Gardos, G. and Penniston, J. T. (1991) Calmodulin-binding domains from isozymes of the plasma membrane $\mathrm{Ca}^{2+}$ pump have different regulatory properties. J. Biol. Chem. 266, 8952-8956

4 Verma, A. K., Filoteo, A. G., Stanford, D. R., Wieben, E. D., Penniston, J. T., Strehler, E. E., Fischer, R., Heim, R., Vogel, G., Matthews, S. et al. (1988) Complete primary structure of a human plasma membrane $\mathrm{Ca}^{2+}$ pump. J. Biol. Chem. 263 , 14152-14159

5 Toyoshima, C., Nakasako, M., Nomura, H. and Ogawa, H. (2000) Crystal structure of the calcium pump of sarcoplasmic reticulum at $2.6 \AA$ resolution. Nature (London) 405, 647-655

6 Enyedi, Á., Elwess, N. L., Filoteo, A. G., Verma, A. K., Paszty, K. and Penniston, J. T. (1997) Protein kinase $C$ phosphorylates the forms of plasma membrane $\mathrm{Ca}^{2+}$ pump isoforms 2 and 3 and prevents binding of calmodulin. J. Biol. Chem. 272, 27525-27528

7 Enyedi, Á., Verma, A. K., Filoteo, A. G. and Penniston, J. T. (1996) Protein kinase C activates the plasma membrane $\mathrm{Ca}^{2+}$ pump Isoform $4 \mathrm{~b}$ by phosphorylation of an inhibitory region downstream of the calmodulin-binding domain. J. Biol. Chem. 271, 32461-32467

8 Caride, A. J., Elwess, N. L., Verma, A. K., Filoteo, A. G., Enyedi, Á., Bajzer, Z. and Penniston, J. T. (1999) The rate of activation by calmodulin of isoform 4 of the plasma membrane $\mathrm{Ca}^{2+}$ pump is slow and is changed by alternative splicing. J. Biol. Chem. 274, 35227-35232

9 Carafoli, E. (1994) Biogenesis: Plasma membrane calcium ATPase: 15 years of work on the purified enzyme. FASEB J. 8, 993-1002

10 Enyedi, Á., Verma, A. K., Heim, R., Adamo, H. P., Filoteo, A. G., Strehler, E. E. and Penniston, J. T. (1994) The $\mathrm{Ca}^{2+}$ affinity of the plasma membrane $\mathrm{Ca}^{2+}$ pump is controlled by alternative splicing. J. Biol. Chem. 269, 41-43

11 Hilfiker, H., Guerini, D. and Carafoli, E. (1994) Cloning and expression of isoform 2 of the human plasma membrane $\mathrm{Ca}^{2+}$ ATPase. Functional properties of the enzyme and its splicing products. J. Biol. Chem. 269, 26178-26183

12 Filoteo, A. G., Enyedi, Á., Verma, A. K., Elwess, N. and Penniston, J. T. (2000) Plasma membrane $\mathrm{Ca}^{2+}$ pump isoform $3 f$ is weakly stimulated by calmodulin. J. Biol. Chem. 275, 4323-4328

Received 15 November 2000/19 February 2001; accepted 2 March 2001 